\title{
Star formation in the Vela molecular ridge
}

\section{Large scale mapping of cloud $\mathrm{D}$ in the $\mathrm{mm}$ continuum ${ }^{\star}$}

\author{
F. Massi ${ }^{1}$, M. De Luca ${ }^{2,3}$, D. Elia ${ }^{4}$, T. Giannini ${ }^{3}$, D. Lorenzetti ${ }^{3}$, and B. Nisini ${ }^{3}$ \\ 1 INAF - Osservatorio Astrofisico di Arcetri, Largo E. Fermi 5, 50125 Firenze, Italy \\ e-mail: fmassi@arcetri.astro.it \\ 2 Dipartimento di Fisica, Università degli studi di Roma Tor Vergata, via della Ricerca Scientifica 1, 00133 Roma, Italy \\ 3 INAF - Osservatorio Astronomico di Roma, via Frascati 33, 00040 Monteporzio Catone, Roma, Italy \\ e-mail: [deluca;giannini; dloren; bruni] amporzio.astro.it \\ 4 Dipartimento di Fisica, Università del Salento, CP 193, 73100 Lecce, Italy \\ e-mail: eliad@le.infn.it
}

Received 25 September 2006 / Accepted 9 February 2007

\section{ABSTRACT}

\begin{abstract}
Context. The Vela molecular ridge is one of the nearest intermediate-mass star forming regions, located within the galactic plane and outside the solar circle. Cloud D, in particular, hosts a number of small embedded young clusters.

Aims. We present the results of a large-scale map in the dust continuum at $1.2 \mathrm{~mm}$ of a $\sim 1^{\circ} \times 1^{\circ}$ area within cloud D. The main aim of the observations was to obtain a complete census of cluster-forming cores and isolated (both high- and low-mass) young stellar objects in early evolutionary phases.

Methods. The bolometer array SIMBA at SEST was used to map the dust emission in the region with a typical sensitivity of $\sim 20 \mathrm{mJy} /$ beam. This allows a mass sensitivity of $\sim 0.2 M_{\odot}$. The resolution is $24^{\prime \prime}$, corresponding to $\sim 0.08$ pc, roughly the radius of a typical young embedded cluster in the region. The continuum map is also compared to a large scale map of $\mathrm{CO}(1-0)$ integrated emission.

Results. Using the CLUMPFIND algorithm, a robust sample of 29 cores has been obtained, spanning the size range $0.03-0.25$ pc and the mass range $0.4-88 M_{\odot}$. The most massive cores are associated both with red IRAS sources and with embedded young clusters, and coincide with $\mathrm{CO}(1-0)$ integrated emission peaks. The cores are distributed according to a mass spectrum $\sim M^{-\alpha}$ and a massversus-size relation $\sim D^{x}$, with $\alpha \sim 1.45-1.9$ and $x \sim 1.1-1.7$. They appear to originate in the fragmentation of gas filaments seen in $\mathrm{CO}(1-0)$ emission and their formation is probably induced by expanding shells of gas. The core mass spectrum is flatter than the Initial Mass Function of the associated clusters in the same mass range, suggesting further fragmentation within the most massive cores. A threshold $A_{\mathrm{V}} \sim 12 \mathrm{mag}$ seems to be required for the onset of star formation in the gas.
\end{abstract}

Key words. ISM: clouds - ISM: individual objects: Vela molecular ridge - ISM: dust, extinction - stars: formation - submillimeter

\section{Introduction}

$\mathrm{CO}(1-0)$ is the best tracer of molecular gas and the most efficient transition to map large sky areas, but it fails to probe the densest molecular cores. This is due both to its large optical depth and to the fact that $\mathrm{CO}$ freezes onto dust grains at the highest densities. However, the availability of large arrays of submillimetre/millimetre bolometers has recently provided a means to carry out large surveys of entire star forming regions searching for protostellar and starless cores with high sensitivity (see, e.g., Motte et al. 1998, 2001; Johnstone et al. 2000; Kerton et al. 2001; Mitchell et al. 2001; Reid \& Wilson 2005; Mookerjea et al. 2004; Hatchell et al. 2005).

All these studies testify to the need to obtain as completely as possible a census of the youngest stellar population down to the lowest masses, and to perform accurate studies of the environment where young stars form. This is particularly true for massive star forming regions, since they usually are farther away than low-mass star forming regions, with obvious problems in terms of resolution. In this respect, the Vela molecular

* Based on observations collected at the European Southern Observatory, La Silla, Chile, program 71.C-0088. ridge (VMR) has proved to be one of the most interesting targets. It is the nearest giant molecular cloud complex hosting massive (although mostly intermediate-mass) star formation after Orion, but, unlike Orion, it is located within the galactic plane, where most of star formation in the Galaxy occurs. Its distance is probably within a factor 2 of that of Orion ( $700 \mathrm{pc}$ vs. $450 \mathrm{pc})$, so the losses in terms of resolution and sensitivity are not very large and are partly compensated by the bigger observable area with the same instrumental field of view. Also, one can in this manner study many different star forming sites with the same observational and environmental biases.

The VMR was first mapped in the ${ }^{12} \mathrm{CO}(1-0)$ transition with low resolution $\left(\sim 30^{\prime}\right)$ by Murphy \& May (1991). They subdivided it into four main regions, named A, B, C and D. Yamaguchi et al. (1999) presented new higher-resolution $\left(\sim 8^{\prime}\right)$ maps of the VMR in the ${ }^{12} \mathrm{CO}(1-0)$ and ${ }^{13} \mathrm{CO}(1-0)$ transitions, better evidencing the complex structure. The issue of distance was discussed by Liseau et al. (1992), who found that clouds A, B and D are at $700 \pm 200$ pc. Liseau et al. (1992) and Lorenzetti et al. (1993) obtained the first data on young stellar objects in the region measuring the spectral energy distribution (SED) for the associated IRAS sources. Massi et al. (1999, 2000, 2003) 
subsequently found that the IRAS sources with $L_{\text {bol }}>10^{3} L_{\odot}$ are associated with young embedded clusters.

Vela D appears to be the most efficient of the VMR clouds in producing young embedded clusters. A look at Fig. 1 of Yamaguchi et al. (1999) shows that the region consists of more than one cloud; that located at $\ell=264^{\circ}, b=0^{\circ}$ contains at least 5 embedded clusters (see Fig. 1 of Massi et al. 2003), all studied by Massi et al. (2000, 2003, 2006). With the aim of investigating the mechanisms leading to this high efficiency, and the star formation history in general, in 1999 we decided to make large scale maps of the cloud at $\mathrm{mm}$ wavelengths with the SEST. Observations in ${ }^{12} \mathrm{CO}(1-0)$ and ${ }^{13} \mathrm{CO}(2-1)$ are presented in Elia et al. (2007). In 2003, the same region was mapped in the 1.2-mm continuum emission with the bolometer array SIMBA. The SEST beam, 24", i.e. $\sim 0.08 \mathrm{pc}$ at $700 \mathrm{pc}$, matches, or is smaller than, the typical spatial size of cluster-forming cores and that of the embedded star clusters in the region (see Massi et al. 2006). A small subset of these data has already been published in Giannini et al. (2005). This paper presents the full results of the SIMBA observations. In Sect. 2, observations and data reduction are described. Section 3 reports the most significant results, that are then discussed in Sect. 4, and Sect. 5 summarizes our conclusions.

\section{Observations and data reduction}

$\mathrm{A} \sim 1^{\circ} \times 1^{\circ}$ region of Vela $\mathrm{D}$ was observed in the $1.2-\mathrm{mm}$ continuum between May 23-26, 2003, using the 37-channel bolometer array SIMBA (Nyman et al. 2001) at the Swedish-ESO Submillimetre Telescope (SEST) sited in La Silla, Chile. At this wavelength, the beam HPBW is $24^{\prime \prime}$. A total of 17 sky areas $18^{\prime} \times 14^{\prime}$ (azimuth $\times$ elevation) in size were mapped in the fast scanning mode, with a scanning speed of $80^{\prime \prime} \mathrm{s}^{-1}$. Each map was repeated 3 to 4 times, except for 3 marginal regions. We always performed a skydip and pointing check every $\sim 2 \mathrm{~h}$, while the focus was checked at the beginning of each observing run and at sunset. The pointing was better than $\sim 5^{\prime \prime}$. The zenith atmospheric opacities remained in the ranges 0.166-0.244 (May 26) and 0.263-0.317 (May 23).

All data were reduced with MOPSI ${ }^{1}$ according to the SIMBA Observer's Handbook (2003). The steps are summarized in Chini et al. (2003). All the available maps for each of the 17 areas were first coadded yielding 17 images that, once calibrated (see below) were mosaiced together. Since some of the images partially overlap, in those cases the reduction steps were repeated while mosaicing in order to search for faint sources emerging from the areas with a longer effective integration time. The final large-scale map is shown in Fig. 1. The rms is in the range $14-40 \mathrm{mJy} /$ beam, but is $\sim 20 \mathrm{mJy} /$ beam (or less) over most of the map.

Unfortunately, the only planet up during the observations was Jupiter, which was mapped once per day. Since it is resolved by the SEST beam, we decided then to calibrate our 1.2-mm data also using observations of Uranus carried out soon before and after each of our observing runs. We derived the conversion factor from counts to $\mathrm{mJy} / \mathrm{beam}$ using different methods, e.g., by fitting a Gaussian to Uranus, by performing aperture photometry of the calibrator using different apertures, by correcting the photometry of Jupiter for source coupling and the error beam. We obtained

${ }^{1}$ MOPSI is a software package for infrared, millimetre and radio data reduction developed and regularly upgraded by R. Zylka. conversion factors within the range $55-75 \mathrm{mJy} / \mathrm{beam}_{\text {counts }}{ }^{-1}$, depending on the adopted method, but remarkably, each method provides a value that remains constant within a $20 \%$ (most often, within a $10 \%$ ) throughout the whole observing period (i.e., one week).

Hence, we adopted the canonical value of $65 \mathrm{mJy} / \mathrm{beam}$ counts $^{-1}$ for all runs but the last, which always appears to approximate the measured values within $\sim 10 \%$. Since all our estimates of the conversion factor exhibit a small drop during the last run (May 26), in this case we adopted a value of $60 \mathrm{mJy} / \mathrm{beam}$ counts $^{-1}$. Anyway, this choice does not affect the results, since only few faint sources were detected during this run. To check our data for consistency, we performed some aperture photometry on a few sources using MOPSI. An example is the region including IRS16 (here we will use the designation adopted by Liseau et al. 1992 for the IRAS sources common to their list) which was observed both on May 23 and 24. The flux from the most intense source is found to be the same within $\sim 3 \%$, confirming the excellent stability of the instrumental setting and atmospheric conditions during the observations. In order to compare our data with the single pointing measurements reported by Massi et al. (1999, 2003), we roughly simulated single pointings by selecting apertures with a diameter equal to the beam HPBW and centred on the positions given by those authors. For the eight common sources observed during our run, we find flux densities within $36 \%$ of those measured by Massi et al. (1999, 2003); in one case, the flux density value of Massi et al. (1999) is within $39 \%$ of ours. However, from a check to the records of the observations listed in Massi et al. (1999) it seems that the single pointings are significantly affected by the selected throw.

A much more significant test can be performed by considering different photometry of sources observed in the same mode. Faúndez et al. (2004) mapped a sample of fields, which includes IRS17, with SIMBA in fast scanning mode. They find a flux of $6.1 \mathrm{Jy}$ for IRS17, while we find a total flux of $8.6 \mathrm{Jy}$ (see Giannini et al. 2005). However, we achieved an rms of $\sim 15 \mathrm{mJy} /$ beam, less than that of Faúndez et al. 2004, implying we might have recovered more low-level emission. Fitting a Gaussian to the source, we obtained a size of $44^{\prime \prime}$ (same as Faúndez et al. 2004) and a flux of $5.5 \mathrm{Jy}$, within $\sim 10 \%$ of that measured by Faúndez et al. (2004). Beltrán et al. (2006) observed four of our sources in their survey of massive protostar candidates with SIMBA, namely IRS16, IRS20 and IRS21 (plus IRS18, observed in our run but located outside the target area, thus not discussed here). They used CLUMPFIND to deconvolve the emission into clumps as we also did (see Sect. 3 ). Since their maps are noisier and less sampled than ours, the identified clumps are slightly different from the ones we detected. Hence, where the number of clumps per source differs, we summed together the emission such as to compare the same emitting areas. In two cases (IRS18 and IRS20), the two sets of fluxes agree within $\sim 15 \%$. As for IRS16 and IRS21, the fluxes measured by Beltrán et al. (2006) are lower and within $\sim 50 \%$ of ours. We carried out aperture photometry with MOPSI on both sets of data, and found that the rms of the four maps by Beltrán et al. (2006) is twice as high as ours. Their scanned areas are much smaller than ours, also, and when using a small aperture around the most intense peaks, the fluxes from their frames are found to be within $\sim 25 \%$ of ours both for IRS16 and IRS21, confirming that probably we recover more low level emission than Beltrán et al. (2006). We conclude that our flux calibration is accurate in general to within $\sim 20 \%$. 


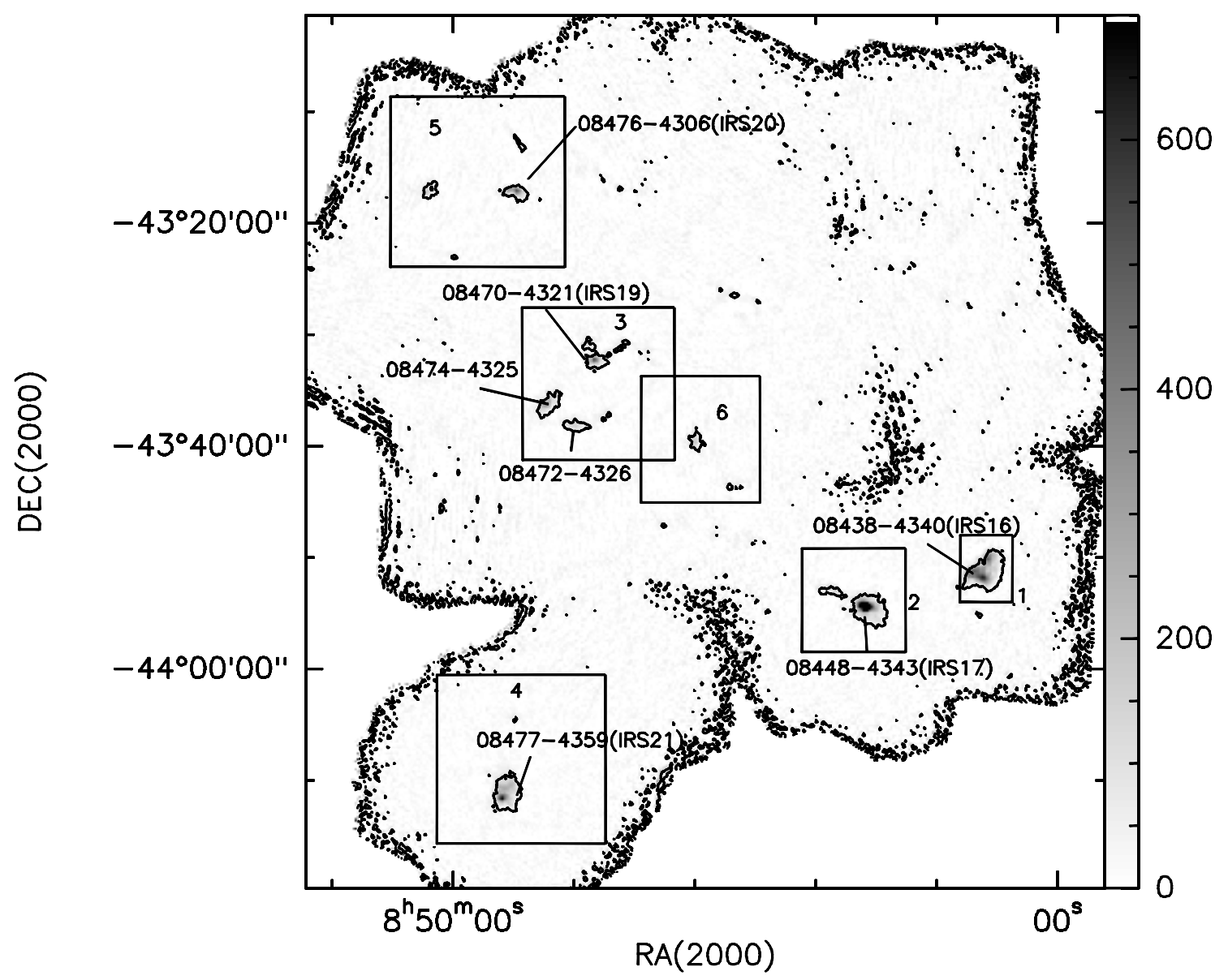

Fig. 1. SIMBA 1.2-mm continuum map of cloud D (greyscale). The scale (in mJy/beam) is indicated through the bar on the right and the contour at $60 \mathrm{mJy} /$ beam $(\sim 3 \sigma)$ is also drawn. The red IRAS sources coincident with the most intense features are labelled (along with the designation adopted by Liseau et al. 1992). Six areas including the main $\mathrm{mm}$ sources are enclosed within boxes and numbered, and are shown zoomed-in in the following figures.

\section{Results}

\subsection{Dense cores}

Figure 1 shows a number of clumps throughout the region, most of which are close to red IRAS sources. Among the young star clusters studied by Massi et al. (2000, 2003, 2006), those that are located within the boundaries of the observed area clearly coincide with the brightest $\mathrm{mm}$ emission sources.

The map has been scanned, searching for dust condensations, using a two-dimensional version of the tool CLUMPFIND, developed by Williams et al. (1994), capable of recognizing connected regions of emission with arbitrary shapes (hereafter cores), starting from the peaks of intensity and going down to the lowest contour levels depending on the rms of the data.

In order to reduce the influence of the variations in the rms noise over the map, we divided the observed region into ten smaller sub-regions. Then, we applied CLUMPFIND to each of these areas using a local value of the rms, as to properly set the two input parameters: the lowest contour level, $I_{\min }$, which acts as a detection threshold, and the intensity level increment, $\Delta I$, used to separate resolved components located within the same emission region. We in practise used $I_{\min }=3 \mathrm{rms}$ and $\Delta I=2 \mathrm{rms}$ in order to be conservative and to obtain a separation between substructures which seems reasonable on a visual inspection.
The varying rms, and the different input parameters chosen for each sub-region, do affect the sensitivity over the map, but ultimately they only change the "global" completeness limit (estimated in Sect. 4). Above this completeness limit, we do not expect our source statistics to be significantly biased.

The CLUMPFIND output consists of a sample of about 50 cores for which we derived the sizes by assuming a distance of 700 pc. From the original output we selected a robust sample of 29 cores (listed in Table 1) which fulfil the criterion of having a size (before beam deconvolution) greater than, or equal to, the SIMBA HPBW. The sample does not include those detections (although all are above $3 \mathrm{rms}$ ) that are revealed by CLUMPFIND, but "under-resolved" because their size is less than the SIMBA beam, before deconvolution. For completeness, we also list coordinates and peak intensity of these detections (estimates of sizes cannot be given) in Table 2. Most of them are small components $\left(\approx 1 M_{\odot}\right)$ of large complex structures, but some (including isolated ones) appear in some way linked to the star formation activity (e.g. coincidence with IRAS/MSX point sources, $\mathrm{CO}$ peaks and/or evidence of $\mathrm{H}_{2}$ emission), although we cannot exclude they are artefacts of the finding algorithm. In particular, umms1 is a relatively high-mass core (but being located within a quite noisy area) and coincides with a $\mathrm{CO}(1-0)$ peak at the border of the line map (hence only partially mapped). However, all these detections deserve dedicated observations with higher sensitivity in order to confirm them, to clarify their nature and to give reasonable estimates of their masses 
Table 1. Dust cores found by CLUMPFIND: the robust sample.

\begin{tabular}{ccccccc}
\hline \hline $\begin{array}{c}\text { Core } \\
\text { designation }\end{array}$ & RA(J2000) & Dec(J2000) & $\begin{array}{c}\text { Deconvolved } \\
\text { size } \\
(\mathrm{pc})\end{array}$ & $\begin{array}{c}\text { Peak } \\
\text { flux } \\
\text { (mJy/beam) }\end{array}$ & $\begin{array}{c}\text { Integrated } \\
\text { flux } \\
(\mathrm{Jy})\end{array}$ & $\begin{array}{c}\text { Mass } \\
\left(M_{\odot}\right)\end{array}$ \\
\hline MMS1 & $8: 45: 33.4$ & $-43: 50: 20.1$ & 0.19 & 501 & 3.17 & 32 \\
MMS2 & $8: 45: 34.8$ & $-43: 52: 04.1$ & 0.17 & 668 & 3.70 & 37 \\
MMS3 & $8: 45: 40.1$ & $-43: 51: 32.3$ & 0.19 & 544 & 3.59 & 36 \\
MMS4 & $8: 46: 34.6$ & $-43: 54: 36.0$ & 0.25 & 1702 & 8.79 & 88 \\
MMS5 & $8: 46: 49.4$ & $-43: 53: 08.2$ & 0.09 & 155 & 0.28 & 3 \\
MMS6 & $8: 46: 52.3$ & $-43: 52: 59.9$ & 0.24 & 121 & 0.20 & 2 \\
MMS7 & $8: 47: 58.8$ & $-43: 39: 47.9$ & 0.08 & 180 & 0.43 & 4 \\
MMS8 & $8: 48: 39.4$ & $-43: 31: 23.9$ & 0.02 & 77 & 0.08 & 0.8 \\
MMS9 & $8: 48: 43.0$ & $-43: 31: 48.0$ & 0.08 & 87 & 0.15 & 1.5 \\
MMS10 & $8: 48: 43.0$ & $-43: 37: 08.0$ & 0.03 & 83 & 0.13 & 1.3 \\
MMS11 & $8: 48: 45.1$ & $-43: 37: 40.1$ & 0.06 & 82 & 0.12 & 1.2 \\
MMS12 & $8: 48: 49.0$ & $-43: 32: 28.0$ & 0.13 & 617 & 1.77 & 18 \\
MMS13 & $8: 48: 49.7$ & $-43: 33: 15.9$ & 0.05 & 69 & 0.04 & 0.4 \\
MMS14 & $8: 48: 51.1$ & $-43: 31: 08.1$ & 0.08 & 117 & 0.12 & 1.8 \\
MMS15 & $8: 48: 52.6$ & $-43: 30: 28.1$ & 0.03 & 91 & 0.12 & 1.2 \\
MMS16 & $8: 48: 53.3$ & $-43: 31: 00.1$ & 0.05 & 163 & 0.23 & 2.3 \\
MMS17 & $8: 48: 57.8$ & $-43: 38: 28.0$ & 0.15 & 156 & 0.76 & 7.6 \\
MMS18 & $8: 49: 03.6$ & $-43: 38: 12.1$ & 0.09 & 105 & 0.28 & 2.8 \\
MMS19 & $8: 49: 08.9$ & $-43: 35: 48.1$ & 0.11 & 126 & 0.38 & 3.8 \\
MMS20 & $8: 49: 11.8$ & $-43: 35: 24.0$ & 0.09 & 126 & 0.19 & 1.9 \\
MMS21 & $8: 49: 13.2$ & $-43: 36: 28.1$ & 0.17 & 345 & 1.76 & 18 \\
MMS22 & $8: 49: 27.4$ & $-43: 17: 08.2$ & 0.09 & 567 & 1.32 & 13 \\
MMS23 & $8: 49: 29.5$ & $-44: 04: 36.1$ & 0.06 & 71 & 0.14 & 1.4 \\
MMS24 & $8: 49: 31.0$ & $-43: 17: 08.2$ & 0.06 & 421 & 0.76 & 7.6 \\
MMS25 & $8: 49: 31.0$ & $-44: 10: 44.1$ & 0.21 & 258 & 1.70 & 17 \\
MMS26 & $8: 49: 33.8$ & $-44: 10: 59.9$ & 0.20 & 298 & 1.55 & 15 \\
MMS27 & $8: 49: 34.6$ & $-44: 11: 56.0$ & 0.18 & 710 & 2.85 & 28 \\
MMS28 & $8: 50: 09.4$ & $-43: 16: 27.8$ & 0.06 & 118 & 0.20 & 2.0 \\
MMS29 & $8: 50: 12.2$ & $-43: 17: 16.1$ & 0.10 & 177 & 0.51 & 5.1 \\
\hline
\end{tabular}

and sizes. A detailed analysis of these uncertain detections will be presented in a forthcoming paper (De Luca et al. 2007), while in the following we will refer only to the sample of Table 1 . The adopted approach is bound to affect the statistic of the faintest sources, dropping objects that would fall in the robust sample if the sensitivity were higher. But, again, we do not expect major effects above the completeness limit.

The cores' masses have been determined accordingly from the expression of Faúndez et al. (2004), by assuming a gas to dust ratio of 100 , a temperature of $30 \mathrm{~K}$ and a dust opacity $k_{1.3}=0.5 \mathrm{~cm}^{2} \mathrm{~g}^{-1}$ at $1.3 \mathrm{~mm}$. We adopted the same $k_{1.3}$ as in Motte et al. (1998) and Testi \& Sargent (1998); however, values as high as $1 \mathrm{~cm}^{2} \mathrm{~g}^{-1}$ are possible, typical of very dense regions $\left(n_{\mathrm{H}}>10^{7} \mathrm{~cm}^{-3}\right.$; Ossenkopf \& Henning 1994), such as, e.g., circumstellar envelopes.

By fitting (emissivity $\epsilon_{\lambda} \sim \lambda^{-1}$ ) the IRAS fluxes at 60 and $100 \mu \mathrm{m}$, Liseau et al. (1992) find temperatures in the range 30-43 K for IRS 17, 18, 19, 20 and 21. Faúndez et al. (2004) derive a typical temperature of $32 \mathrm{~K}$ for the cold dust component by a two-components fit to the SED's in their sample of southern high-mass star forming regions. Beltrán et al. (2006) find a mean dust temperature of $28 \mathrm{~K}$ by fitting the SED's in their sample of southern massive protostar candidates longward of $60 \mu \mathrm{m}$. Hence, we adopted the canonical value of $30 \mathrm{~K}$ for all the cores. Nevertheless, some of the cores may be colder prestellar cores, and decreasing the dust temperature to $15 \mathrm{~K}$ would increase the derived masses by a factor 2.5. Actually, an external heating could drive the temperature of pre-stellar cores towards higher values. Evidence of heating resulting from a strong external radiation field has been found for protostellar cores in Orion by Jørgensen et al. (2006). However, the presence of energetic sources in the mapped region of Vela-D is still controversial (Lorenzetti et al. 1993; Elia et al. 2007).

The deconvolved sizes and the masses of the cores listed in Table 1 range from 0.03 to $0.25 \mathrm{pc}$ and 0.4 to $88 M_{\odot}$ respectively, while their spatial distribution exhibits a high degree of clustering. The derived sizes span the interval from the observed diameter of pre-stellar cores in cluster-forming regions to that of cluster-forming cores (or isolated pre-stellar cores). The achieved typical sensitivity of $20 \mathrm{mJy} /$ beam translates into a point source mass sensitivity (at a $1 \sigma$ level) of $\sim 0.2 M_{\odot}$, using the above adopted temperature, opacity and distance. However, see the next section for an assessment of the mass sensitivity to extended sources.

\subsection{Contamination sources of dust emission}

While the effect of non-uniform dust temperature and opacity throughout the sample will be discussed in the next section, the other possible causes of error in the derived masses are contamination by: i) line emission; ii) optically-thin free-free emission; iii) optically-thick free-free emission, and iv) synchrotron radiation. In the following, we discuss each of the contamination sources.

The line that can mostly affect the detected signal is ${ }^{12} \mathrm{CO}(2-1)$. We estimated its contribution using the relation given by Braine et al. (1995), adapted to our instrumental parameters, and our observations of $\mathrm{CO}(1-0)$. Since $\mathrm{CO}(1-0)$ and $\mathrm{CO}(2-1)$ are optically thick, assuming the same excitation temperature yields a $\mathrm{CO}(2-1)$ to $\mathrm{CO}(1-0)$ ratio $\leq 1$. Due to the larger beam at the frequency of $\mathrm{CO}(1-0)$ with respect to that at $1.2 \mathrm{~mm}$, 
Table 2. Possible dust cores found by CLUMPFIND, but with size less than the SEST beam (before deconvolution).

\begin{tabular}{ccccccc}
\hline \hline $\begin{array}{c}\text { Core } \\
\text { designation }\end{array}$ & RA(J2000) & Dec(J2000) & $\begin{array}{c}\text { Peak } \\
\text { flux } \\
\text { (mJy/beam) }\end{array}$ & $\begin{array}{c}\text { Peak } \\
\text { flux } \\
(\times \text { local rms })\end{array}$ & $\begin{array}{c}\text { Integrated } \\
\text { flux } \\
(\text { Jy })\end{array}$ & Mass \\
\hline umms1 & $8: 46: 25.8$ & $-43: 42: 26.6$ & 310 & 6 & 2.78 & 28 \\
umms2 & $8: 46: 37.2$ & $-43: 18: 34.9$ & 86 & 4 & 0.05 & 0.5 \\
umms3 & $8: 46: 37.2$ & $-43: 19: 55.6$ & 175 & 8 & 0.12 & 1.2 \\
umms4 & $8: 46: 49.0$ & $-43: 20: 27.1$ & 111 & 5 & 0.09 & 0.9 \\
umms5 & $8: 46: 50.4$ & $-43: 21: 15.1$ & 90 & 4 & 0.05 & 0.5 \\
umms6 & $8: 46: 56.6$ & $-43: 53: 07.1$ & 97 & 6 & 0.16 & 1.6 \\
umms7 & $8: 47: 28.5$ & $-43: 27: 00.4$ & 75 & 4 & 0.04 & 0.4 \\
umms8 & $8: 47: 37.3$ & $-43: 43: 40.1$ & 71 & 4 & 0.03 & 0.3 \\
umms9 & $8: 47: 39.5$ & $-43: 43: 48.0$ & 65 & 4 & 0.02 & 0.2 \\
umms10 & $8: 47: 41.0$ & $-43: 26: 28.3$ & 84 & 5 & 0.12 & 1.2 \\
umms11 & $8: 47: 42.5$ & $-43: 43: 39.1$ & 139 & 8 & 0.10 & 1.0 \\
umms12 & $8: 47: 46.8$ & $-43: 25: 55.4$ & 79 & 5 & 0.03 & 0.3 \\
umms13 & $8: 47: 55.7$ & $-43: 39: 41.5$ & 81 & 5 & 0.07 & 0.7 \\
umms14 & $8: 47: 57.9$ & $-43: 38: 59.6$ & 110 & 7 & 0.17 & 1.7 \\
umms15 & $8: 48: 02.4$ & $-43: 39: 15.5$ & 107 & 7 & 0.12 & 1.2 \\
umms16 & $8: 48: 15.7$ & $-43: 47: 07.8$ & 92 & 5 & 0.06 & 0.6 \\
umms17 & $8: 48: 23.0$ & $-43: 31: 31.1$ & 63 & 4 & 0.07 & 0.7 \\
umms18 & $8: 48: 26.7$ & $-43: 31: 39.7$ & 66 & 4 & 0.04 & 0.4 \\
umms19 & $8: 48: 33.1$ & $-43: 30: 43.9$ & 83 & 6 & 0.11 & 1.1 \\
umms20 & $8: 48: 35.5$ & $-43: 30: 59.8$ & 83 & 6 & 0.07 & 0.7 \\
umms21 & $8: 48: 36.8$ & $-43: 31: 13.8$ & 76 & 5 & 0.07 & 0.7 \\
umms22 & $8: 48: 36.6$ & $-43: 16: 51.2$ & 84 & 4 & 0.05 & 0.5 \\
umms23 & $8: 49: 24.2$ & $-43: 13: 13.4$ & 95 & 6 & 0.12 & 1.2 \\
umms24 & $8: 49: 27.1$ & $-43: 12: 33.5$ & 86 & 6 & 0.07 & 0.7 \\
umms25 & $8: 49: 27.8$ & $-43: 12: 17.3$ & 83 & 6 & 0.05 & 0.5 \\
umms26 & $8: 49: 59.0$ & $-43: 22: 55.6$ & 91 & 6 & 0.09 & 0.9 \\
\hline
\end{tabular}

by assuming the same main beam temperature for $\mathrm{CO}(2-1)$ and $\mathrm{CO}(1-0)$ we still might slightly underestimate the $\mathrm{CO}(2-1)$ main beam temperature. However, we checked that the line contribution to the integrated flux density is always less than few percent for the cores with $S_{v} \sim 10^{3} \mathrm{mJy}$. Conversely, for the cores with $S_{v} \approx 10^{3} \mathrm{mJy}$, the estimated line contribution is $\sim 10-30 \%(55 \%$ in one case). This is an overestimate, since the integrated emission of $\mathrm{CO}(1-0)$ is $<60 \mathrm{~K} \mathrm{~km} \mathrm{~s}^{-1}$ towards most of these sources and, as can be seen in Fig. 8, at least the integrated emission up to $25 \mathrm{~K} \mathrm{~km} \mathrm{~s}^{-1}$ arises from a distributed structure much larger than the cores' size that has been effectively filtered out by the observing mode of SIMBA (see also Figs. 3 to 7).

Hence, line emission is very likely not to contribute more than $10-20 \%$ of the integrated flux density from the faintest $1.2 \mathrm{~mm}$ sources. These results agree with what extensively discussed by Johnstone et al. 2003; they find that at $850 \mu \mathrm{m}$, only occasionally the continuum becomes contaminated by line emission, including $\mathrm{CO}(3-2)$, in strong sources, although this may be a problem for low continuum flux sources.

Free-free emission from HII regions can also affect the measured flux densities at $1.2 \mathrm{~mm}$. As a first step, we checked that none of the cores' positions coincide with radio sources from the Parkes-MIT-NRAO survey at $4.85 \mathrm{GHz}$ (Griffith \& Wright 1993). Hence, their radio fluxes at $4.85 \mathrm{GHz}$ are lower than the quoted limit of $48 \mathrm{mJy}$. For optically thin free-free sources this translates into $32 \mathrm{mJy}$ at $1.2 \mathrm{~mm}$. Only two of the cores listed in Table 1 exhibit an integrated flux density $<100 \mathrm{mJy}$, whereas for all those with integrated flux density $\sim 10^{2} \mathrm{mJy}$ the value extrapolated from the radio upper limit amount to $<25 \%$ of that measured at $1.2 \mathrm{~mm}$. As shown by Massi et al. (2003), at a distance of $700 \mathrm{pc}$ this upper limit implies no HII regions excited by stars earlier than B1. Although we assumed optically thin emission, this agrees with the limit on the earliest star spectral type set by the observed bolometric luminosities (B1-B2).

The Parkes-MIT-NRAO catalogue lists extended radio emission that, generally, lies a few arcmin from the cores. However, there are some exceptions. Towards IRS 16: MMS1, MMS2 and MMS3 are at a distance of $\sim 1^{\prime}$ from the centre of a radio source (quoted radius $1.1^{\prime}$ ) at $\mathrm{RA}(\mathrm{J} 2000)=08: 45: 36.3$, $\operatorname{Dec}(\mathbf{J} 2000)=-43: 51: 01$. This is the HII region 263.6190.533 (Caswell \& Haynes 1987); the extrapolated flux density (assuming optically thin emission) at $1.2 \mathrm{~mm}$ is $1.6 \mathrm{Jy}$, $15 \%$ of the total integrated flux density of MMS1, MMS2 and MMS3. Assuming the radio emission is uniform over a circle of radius $1.1^{\prime}$, the expected flux density at $1.2 \mathrm{~mm}$ is $52 \mathrm{mJy} /$ beam(SEST), i.e., at a 2-3 $\sigma$ level. Clearly, as can be seen in Fig. 2, the three cores are located at the boundary of the HII region (whose centre almost coincides with the IRAS location and the centre of the radio emission), confirming that they are mostly due to dust thermal radiation. Towards IRS19: MMS19, MMS20 and MMS21 lie 2' from the centre of a radio source (PMNJ0849-4335; semi-major by semi-minor axes $3.8 \times 1.4)$ at $\mathrm{RA}(\mathrm{J} 2000)=08: 49: 22.6, \operatorname{Dec}(\mathrm{J} 2000)=-43: 35: 56$. The flux density extrapolated at $1.2 \mathrm{~mm}$ (assuming optically thin free-free radiation) is $294 \mathrm{mJy}$, with a mean flux density of $2 \mathrm{mJy} /$ beam(SEST), well below our rms This explains why no emission is detected at $1.2 \mathrm{~mm}$ from this radio source. Anyway, the extrapolated total flux density is $\sim 13 \%$ of that measured at $1.2 \mathrm{~mm}$ for the three cores. Finally, towards IRS21: MMS25, MMS26 and MMS27 are located 2-3' from the centre of a radio emission (PMNJ0849-4413; semi-major by semi-minor axes $2.6 \times 1.5)$ at $\mathrm{RA}(\mathrm{J} 2000)=08: 49: 31.2, \operatorname{Dec}(\mathrm{J} 2000)=-44: 13: 47$. The flux density extrapolated at $1.2 \mathrm{~mm}$ (assuming optically thin free-free radiation) is $94 \mathrm{mJy}$, with a mean flux density of $1 \mathrm{mJy} /$ beam(SEST), again well below our rms. In fact, no $\mathrm{mm}$ 


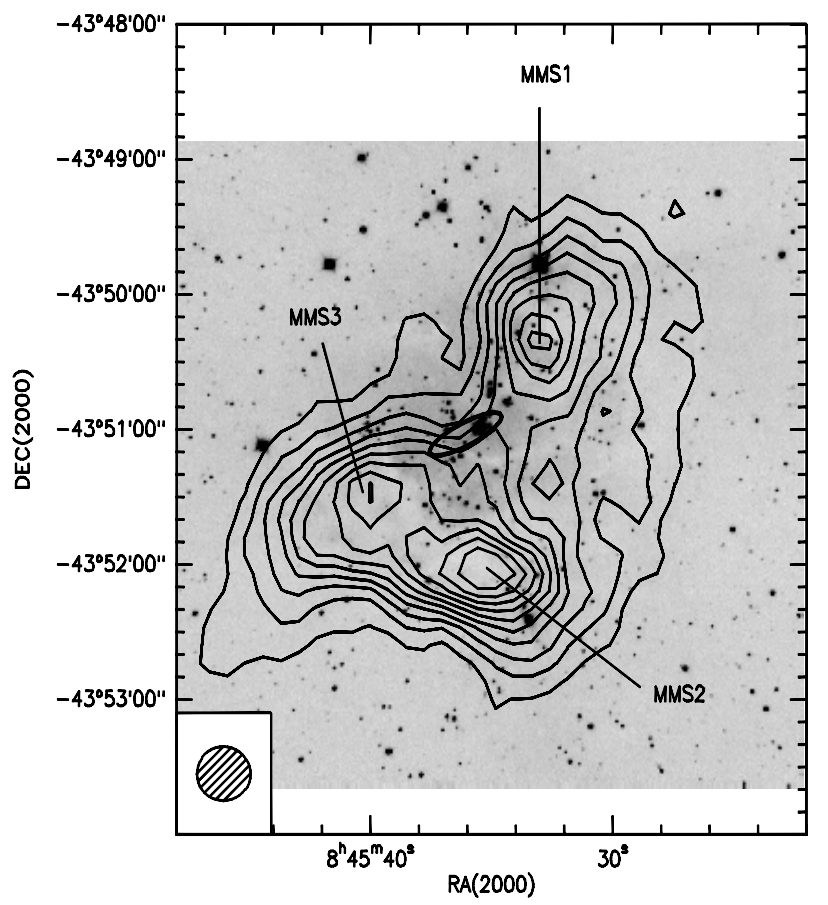

Fig. 2. NTT/SofI image of IRS16 (at $K_{s}$ ), taken on December 2005 , overlaid with a contour map of the 1.2-mm continuum emission (area 1 in Fig. 1). Contours are in steps of $60 \mathrm{mJy} /$ beam $(\sim 3 \sigma)$ from 60 to $600 \mathrm{mJy} /$ beam. The cores are labelled and the IRAS uncertainty ellipse is also drawn. The SEST beam is displayed in the lower left box. The nebulosity surrounding the location of the IRAS source is due to the HII region 263.619-0.533.

emission is detected from this radio source, as well. The total extrapolated flux density is only $<2 \%$ of that measured at $1.2 \mathrm{~mm}$ for the three cores.

Clearly, on the one hand the contamination to the cores' flux densities from optically thin free-free emission is negligible. Synchrotron radiation has an even steeper spectral index than free-free $\left(S_{v} \sim v^{-0.6}\right.$ vs. $\left.S_{v} \sim v^{-0.1}\right)$, so its contribution at $1.2 \mathrm{~mm}$ is expected to be much lower. On the other hand, we cannot exclude some contamination from optically thick freefree emission $\left(S_{v} \sim v^{2}\right.$ up to a turnover frequency depending on the emission measure) arising from ultracompact (or hypercompact) HII regions towards some of the cores. Indeed, as discussed by Massi et al. (2003), some of the IRAS sources towards the cores have the colours of UCHII regions, but the fraction of fields exhibiting other signposts of UCHII regions appears to be very low.

\subsection{Star forming regions}

Clues about the star formation history of the cloud can be derived from the filamentary morphology of the gas (see Elia et al. 2007). As clearly shown in Fig. 8, all $\mathrm{mm}$ cores are aligned along filaments or arcs of molecular gas. IRS16 and IRS17 lie inside one of these arcs, in the south-western part of the map. IRS16 (see Fig. 2) consists of 3 massive cores located around an HII region (roughly $0.4 \mathrm{pc}$ in diameter), suggesting that the expansion of the HII region may have triggered star formation there. On the other hand, IRS17 (see Fig. 3) is part of a filament of mm sources; it contains the most massive core (MMS4) of the whole region, which is slightly elongated in the direction of the larger-scale surrounding $\mathrm{CO}$ arc. Two more cores (MMS5 and MMS6), east of it and again following the molec-

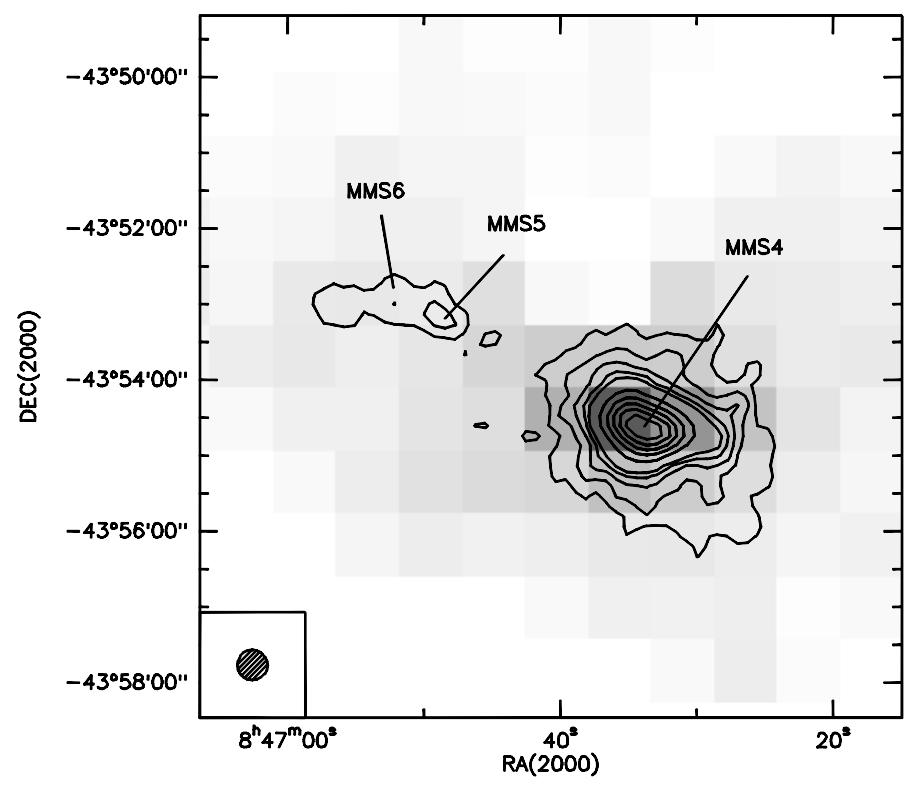

Fig. 3. Contour map of the 1.2-mm continuum emission from the region including IRS17 (area 2 in Fig. 1). Contours are in steps of $60 \mathrm{mJy} / \mathrm{beam}$ $(\sim 3 \sigma)$ from 60 to $300 \mathrm{mJy} / \mathrm{beam}$ and in steps of $240 \mathrm{mJy} / \mathrm{beam}$ from 540 to $1500 \mathrm{mJy} /$ beam. The cores are labelled and the SEST beam is drawn in the lower left box. Underlying, the ${ }^{12} \mathrm{CO}(1-0)$ emission integrated from 0 to $20 \mathrm{~km} \mathrm{~s}^{-1}$ (greyscale, from 30 to $250 \mathrm{~K} \mathrm{~km} \mathrm{~s}^{-1}$ ).

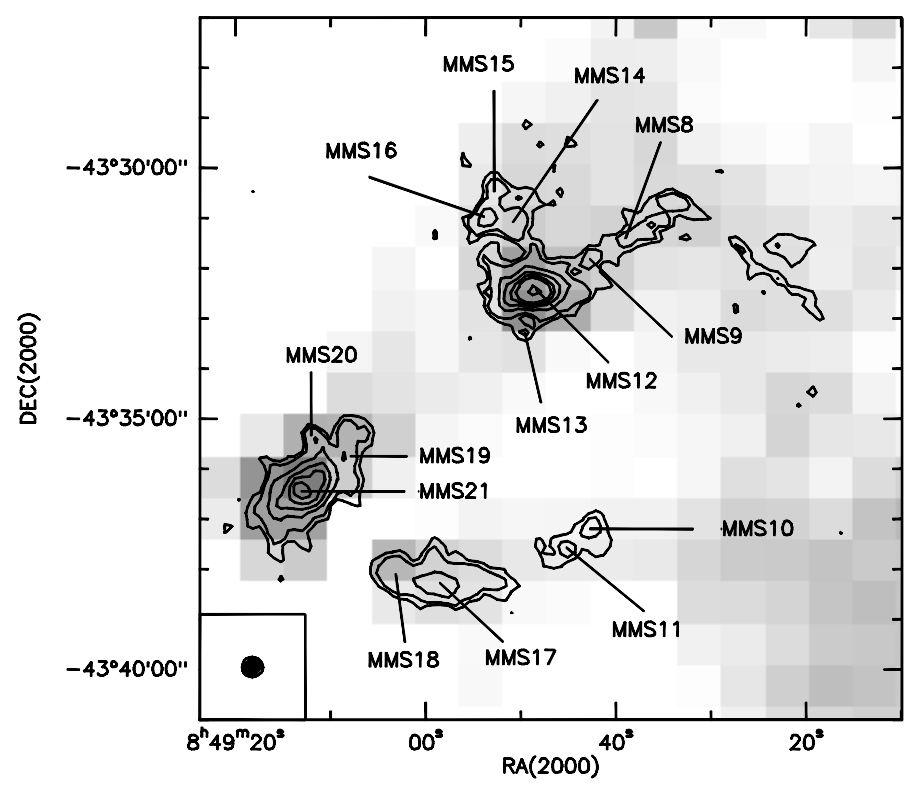

Fig. 4. Contour map of the 1.2-mm continuum emission from the region including IRS19 (area 3 in Fig. 1). Contours are: $40 \mathrm{mJy} /$ beam $(\sim 2 \sigma)$ the lowest one, then in steps of $60 \mathrm{mJy} /$ beam $(\sim 3 \sigma)$ from 60 to $300 \mathrm{mJy} / \mathrm{beam}$ and $540 \mathrm{mJy} / \mathrm{beam}$ the highest one. The cores are labelled and the SEST beam is drawn in the lower left box. Underlying, the ${ }^{12} \mathrm{CO}(1-0)$ emission integrated from 0 to $20 \mathrm{~km} \mathrm{~s}^{-1}$ (greyscale, from 30 to $200 \mathrm{~K} \mathrm{~km} \mathrm{~s}^{-1}$ ).

ular gas orientation, suggest fragmentation of a larger filament. Giannini et al. (2005) showed that MMS4 can be further decomposed into two cores (mmA of $110 M_{\odot}$, and $\mathrm{mmB}$ of $11 M_{\odot}$ ) embedded in more diffuse gas $\left(58 M_{\odot}\right)$. They adopted a smaller dust temperature $(23 \mathrm{~K})$, finding higher masses than ours.

IRS19 (see Fig. 4) is part of a relatively complex structure composed of filaments arranged in a roughly elliptical pattern 


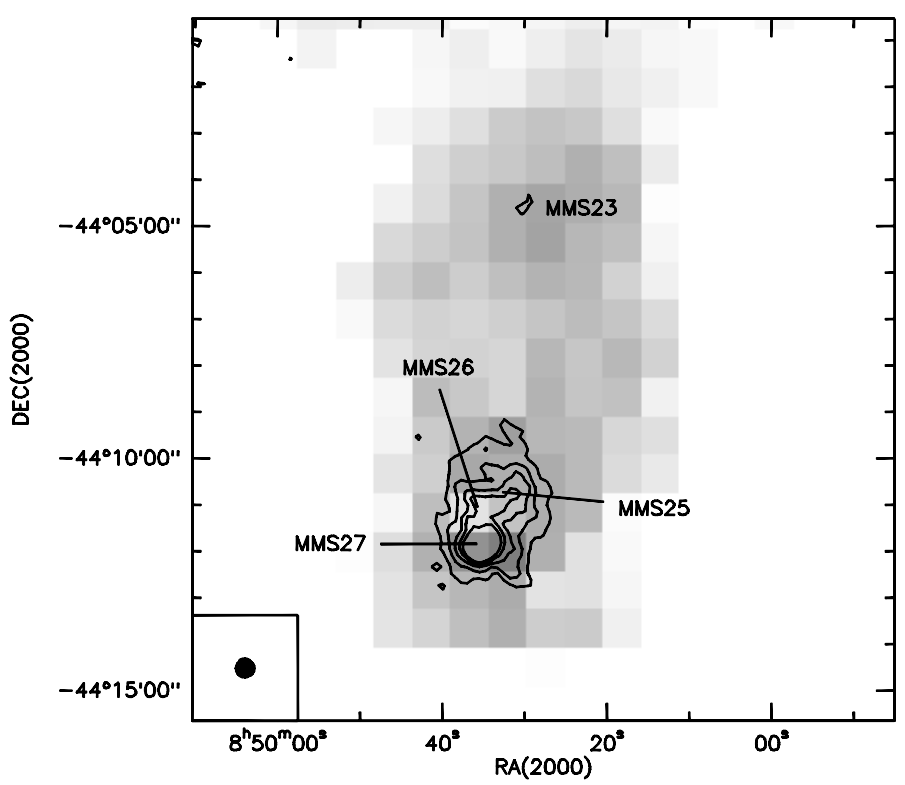

Fig. 5. Contour map of the 1.2-mm continuum emission from the region including IRS21 (area 4 in Fig. 1). Contours are in steps of $60 \mathrm{mJy} /$ beam $(\sim 3 \sigma)$ from 60 to $300 \mathrm{mJy} / \mathrm{beam}$. The cores are labelled and the SEST beam is drawn in the lower left box. Underlying, the ${ }^{12} \mathrm{CO}(1-0)$ emission integrated from 0 to $20 \mathrm{~km} \mathrm{~s}^{-1}$ (greyscale, from 30 to $150 \mathrm{~K} \mathrm{~km} \mathrm{~s}^{-1}$ ).

with a major axis of $\sim 2$ pc. The most massive cores in the pattern (MMS12, MMS17 and MMS21) are all associated with three IRAS sources whose SED is typical of Class I sources (IRAS 08470-4321/IRS19, IRAS 08472-4326, and IRAS 08474-4325, respectively). This structure is reflected in the $\mathrm{CO}(1-0)$ map (Fig. 8) also, suggesting a dynamical origin and a subsequent fragmentation.

IRS21 (see Fig. 5) is the most massive in a chain of cores, also aligned in the same direction as a surrounding filament of molecular gas (i.e., north-south). IRS20 (see Fig. 6), as well, is part of a chain of cores aligned with a surrounding filament of molecular gas (i.e., east-west). Finally, a moderately-massive isolated core lies towards the centre of the molecular cloud (see Fig. 7).

\section{Discussion}

In Fig. 8, we show the $1.2 \mathrm{~mm}$ continuum map overlaid with contours of the map of $\mathrm{CO}(1-0)$ emission integrated from 0 to $20 \mathrm{~km} \mathrm{~s}^{-1}$ (obtained with a beam twice as large as that at $1.2 \mathrm{~mm}$ ). Clearly, the molecular emission arises from filaments and the detected continuum sources lie towards peaks of $\mathrm{CO}(1-0)$ integrated emission within those filaments. This indicates that the mm cores all belong to the Vela D cloud. In particular, the brightest of them are those associated with the red IRAS sources and embedded clusters (IRS16, 17, 19, 20 and 21). The kinematic distances attributed to some of these regions vary in the range 0.6-1.8 kpc (see, e.g., Beltrán et al. 2006; Faúndez et al. 2004); this is due to the structure evidenced by the $\mathrm{CO}(1-$ 0) spectra. Figure 8 shows that most filaments resemble arcs, and velocity-position cuts also give evidence for shell-like structures with differences in velocity of $\sim 5-10 \mathrm{~km}^{-1} \mathrm{~s}^{-1}$. This suggests that the emission actually arises from the same cloud, but that it is fragmented into a number of expanding shells. Hence our choice to attribute to all cores the same distance $(700 \mathrm{pc})$. The issue is discussed in detail in Elia et al. (2007).

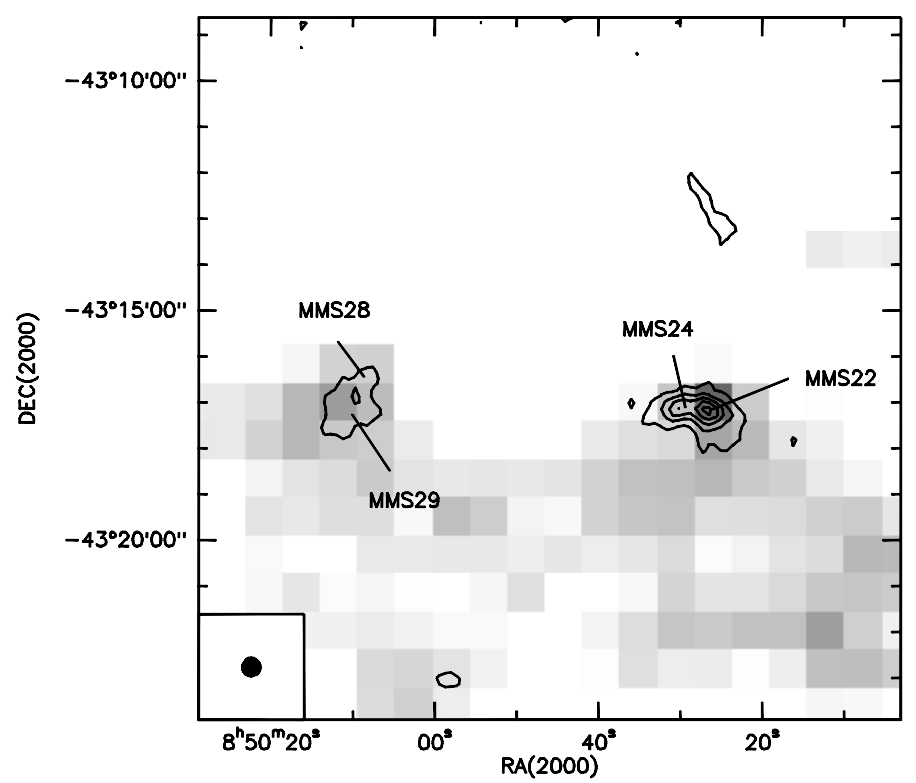

Fig. 6. Contour map of the 1.2-mm continuum emission from the region including IRS20 (area 5 in Fig. 1). Contours are in steps of $120 \mathrm{mJy} /$ beam $(\sim 6 \sigma)$ from 60 to $540 \mathrm{mJy} / \mathrm{beam}$. The cores are labelled and the SEST beam is drawn in the lower left box. Underlying, the ${ }^{12} \mathrm{CO}(1-0)$ emission integrated from 0 to $20 \mathrm{~km} \mathrm{~s}^{-1}$ (greyscale, from 30 to $150 \mathrm{~K} \mathrm{~km} \mathrm{~s}^{-1}$ ).

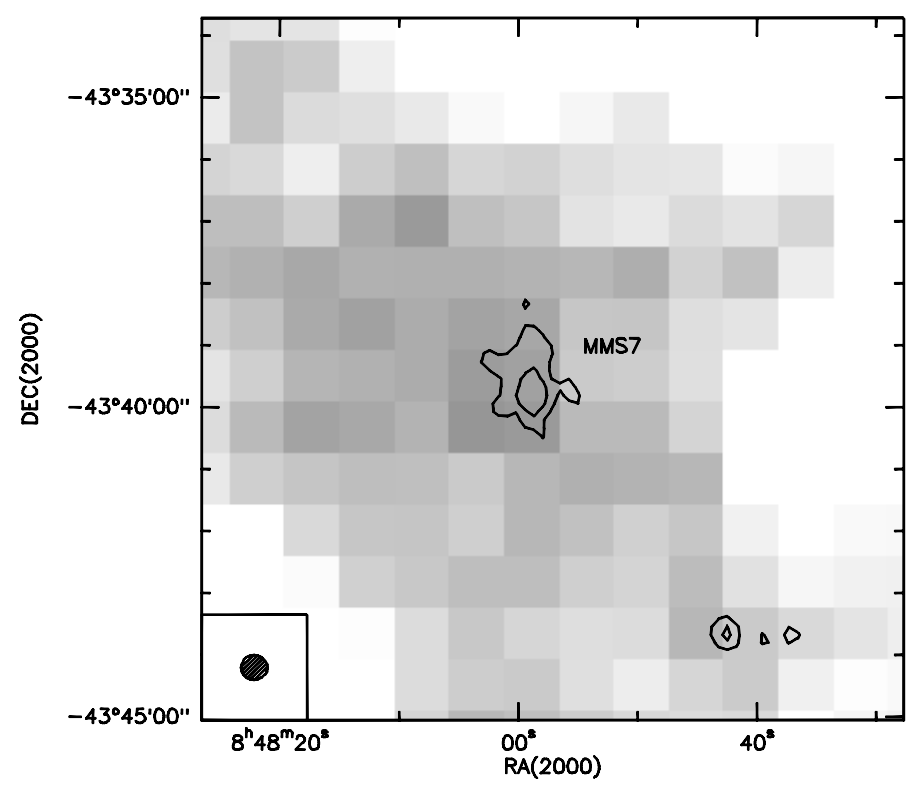

Fig. 7. Contour map of the 1.2-mm continuum emission from the region at the centre of the mapped area (6 in Fig. 1) Contours are in steps of $60 \mathrm{mJy} /$ beam $(\sim 3 \sigma)$ from 60 to $120 \mathrm{mJy} /$ beam. The cores are labelled and the SEST beam is drawn in the lower left box. Underlying, the ${ }^{12} \mathrm{CO}(1-0)$ emission integrated from 0 to $20 \mathrm{~km} \mathrm{~s}^{-1}$ (greyscale, from 30 to $150 \mathrm{~K} \mathrm{~km} \mathrm{~s}^{-1}$ ).

The observed mass-versus-size relationship (uncorrected for beam) is plotted in Fig. 9. The "under-resolved" detections reported in Table 2 fall below the resolution limit given by the beam size (the vertical line in figure).

When assessing the sensitivity of a survey in the mm continuum, one has to take into account the extent of the emitting area. Since the sensitivity limit is a flux density per solid angle, for a given total flux density an extended source is less likely to be detected than a point source. In fact, assuming constant 


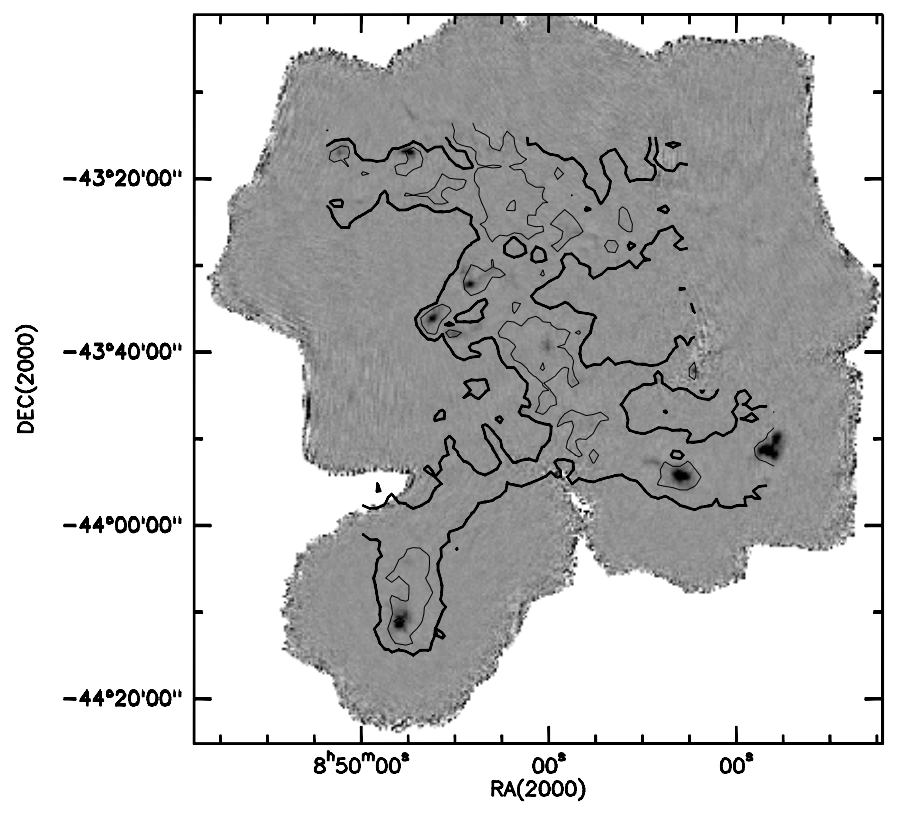

Fig. 8. Map of $\mathrm{CO}(1-0)$ emission from the observed area within cloud D (contours), integrated from 0 to $20 \mathrm{~km} \mathrm{~s}^{-1}$, obtained with a beam of $43^{\prime \prime}$. This is overlaid with the SIMBA $1.2 \mathrm{~mm}$ continuum map (greyscale, beam of $\left.24^{\prime \prime}\right)$. The thick solid line marks the contour at $25 \mathrm{~K} \mathrm{~km} \mathrm{~s}^{-1}$, whereas the light solid line marks the contour at $60 \mathrm{~K} \mathrm{~km} \mathrm{~s}^{-1}$.

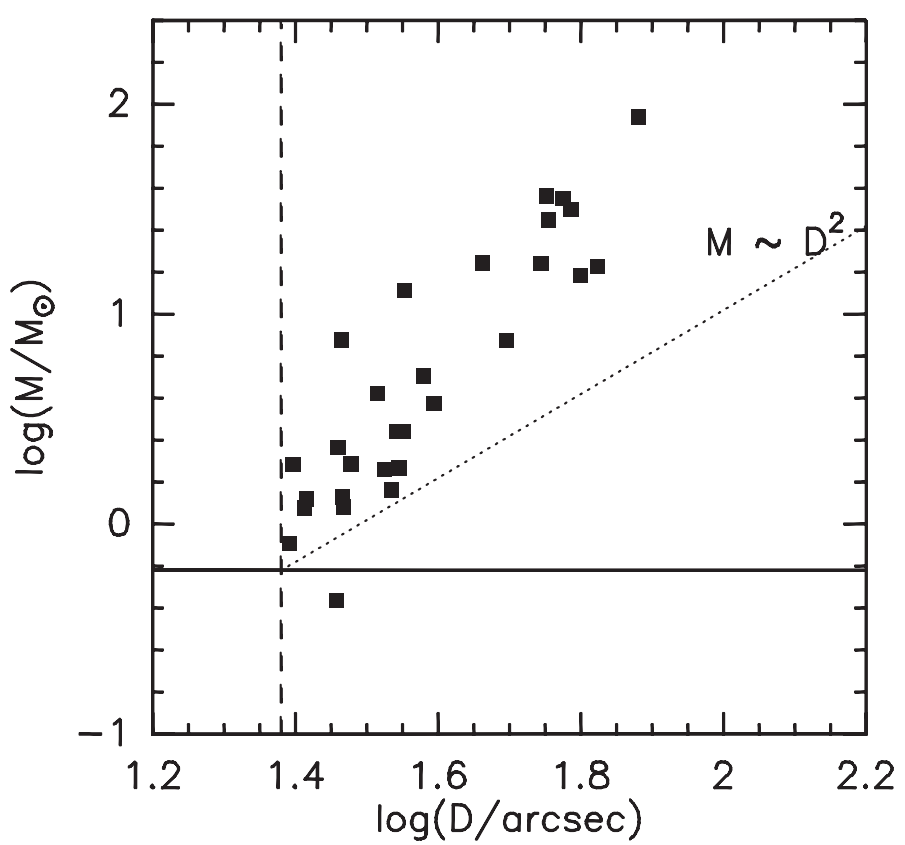

Fig. 9. Cores' mass vs. size (uncorrected for beam). The vertical dashed line marks the SEST beam size, whereas the horizontal solid line shows the $3 \sigma$ detection limit for point sources. A dotted line has also been drawn following a $D^{2}$ relation, i.e., the sensitivity limit to extended sources (see text).

dust opacities and temperatures, the source mass is proportional to the total (i.e., integrated over the solid angle) flux density. Then, the mass sensitivity limit is given by the flux sensitivity per solid angle multiplied by the source area and so increases with increasing areas. This is illustrated in Fig. 9: all sources lie above a line following a $M \sim D^{2}$ relation (where $D$ is the core size), which represents the actual sensitivity limit. Clearly, the

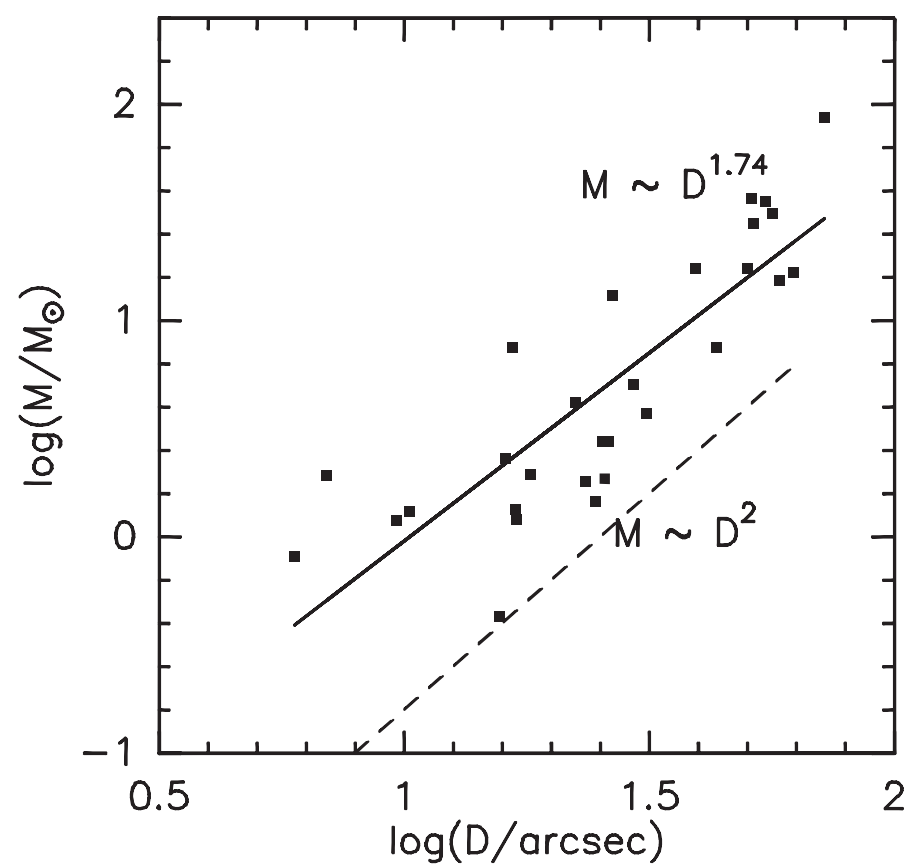

Fig. 10. Cores' mass vs. deconvolved size. The solid line is obtained by a fit $\left(M \sim D^{1.74}\right)$, whereas the dashed line follows a $D^{2}$ relation, i.e., the sensitivity limit to extended sources (see text).

data points outline a steeper dependence of mass vs. size; a linear fit indicates a $M \sim D^{3.7}$ relation. This appears to be intrinsic to the core population, indicating that the sample is likely to be complete at least down to $\sim 1-1.3 M_{\odot}$. A more significant relation is obtained by fitting deconvolved sizes vs. mass (see Fig. 10), yielding $M \sim D^{1.7}$. This is flatter than found for prestar forming regions in the range $0.01-10 \mathrm{pc}$ by, e.g., Heithausen et al. (1998) using CO emission $\left(M \sim D^{2.31}\right)$, but steeper than found for pre-stellar cores by Motte et al. (2001) using submm emission $\left(M \sim D^{1.1}\right)$. Instead, Reid \& Wilson (2005) find $M \sim D^{x}$ with $x \sim 1.5-2.1$ in the high-mass star forming region NGC7538 from submm observations, more in agreement with our result. However, note that 9 out of the 29 sources in our robust sample are smaller than the beam $F W H M$, i.e., their beam-convolved size is $<1.5$ FWHM. Then, the core size may become highly uncertain at the lowest end of the distribution.

The core mass spectrum is shown in Fig. 11. The errorbars are the rms estimated by assuming a Poisson statistic. The distribution appears quite flat and drops abruptly at $\sim 10^{2} M_{\odot}$. A power-law fit to the points above the completeness limit indicates that the mass spectrum follows a relation $\mathrm{d} N / \mathrm{d} M \sim M^{-\alpha}$, with $\alpha=1.45 \pm 0.2\left(\chi^{2}=1.10\right)$. This is shallower than a Salpeter-like Initial Mass Function $(\alpha \sim 2.35)$, being more similar to the mass spectrum of molecular cores in pre-star forming regions (see, e.g., Heithausen et al. 1998). However, Fig. 11 suggests that fitting the core mass function with two powerlaw segments would be more adequate. In this case, we obtain $\alpha=1.20 \pm 0.34\left(\chi^{2}=0.42\right)$ up to $M \sim 40 M_{\odot}$, with a significantly larger $\alpha$ for $M>40 M_{\odot}$. As for (sub-)mm surveys, the derived spectral index roughly agrees with that determined by Kerton et al. (2001) for KR140 between $\sim 0.7-100 M_{\odot}(\alpha=$ 1.49), and by Mookerjea et al. (2004) between 37-16000 $M_{\odot}$ in R106 ( $\alpha \sim 1.5-1.7)$. Reid \& Wilson (2005) also find a flat distribution $(\alpha=0.9 \pm 0.1)$ between $\sim 1-100 M_{\odot}$ in NGC 7538, then steepening to $\alpha=2.0-2.6$. 


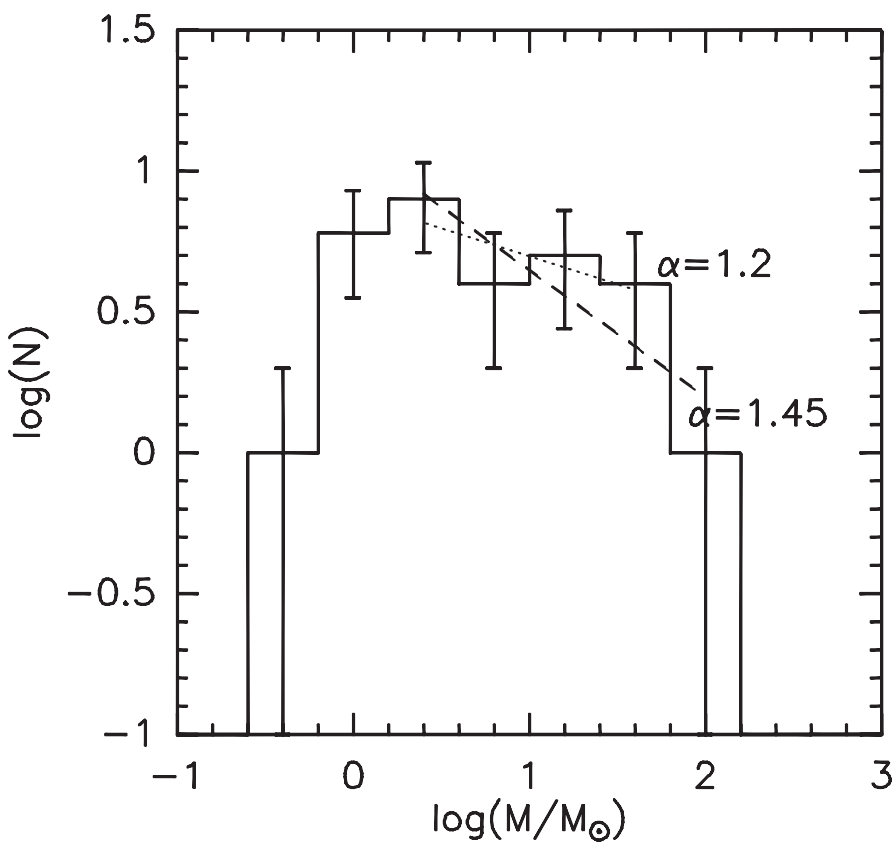

Fig. 11. Mass spectrum of the $\mathrm{mm}$ cores. The errorbars indicate the rms assuming a Poisson statistic. A dashed line shows a $\mathrm{d} N / \mathrm{d} M \sim M^{-1.45}$ relation, a dotted line shows a $\mathrm{d} N / \mathrm{d} M \sim M^{-1.2}$ relation. The estimated completeness limit is $\sim 1-1.3 M_{\odot}$ (see text).

The slope of the mass spectrum and the mass-size relationship are not sensitive to the adopted dust opacity, temperature and distance as long as all cores are characterized by the same mean opacity and temperature and lie at the same distance. As discussed before, both differences in distance and contributions from free-free and synchrotron radiation to the dust thermal emission do appear to be minor issues. Large errors may only be caused by having detected thermal dust emission coming from environments whose temperatures and/or dust opacities differ within the sample. In fact, the identified cores are likely to be a mixture of protostellar envelopes (those coinciding with the red IRAS sources), pre-protostellar cores/Class 0 sources and transient structures. A forthcoming paper (De Luca et al. 2007) will discuss the coincidence between mm cores and NIR/MIR/FIR sources and the nature of the single cores. Here, just to test what would be the effect of gathering together sources with different temperatures and opacities, we have subdivided the robust sample into a subset of objects (10 out of 29) that may be associated with IRAS or MSX sources and a subset of objects unassociated with IRAS or MSX sources (the remaining 19). To the first group we have attributed a temperature of $30 \mathrm{~K}$ and a dust opacity $k_{1.3}=1 \mathrm{~cm}^{2} \mathrm{~g}^{-1}$, whereas to the second group we have attributed a temperature of $15 \mathrm{~K}$ and a dust opacity $k_{1.3}=0.5 \mathrm{~cm}^{2} \mathrm{~g}^{-1}$ (as usually found in the literature for pre-protostellar cores; e.g., Motte et al. 1998, 2001; Nutter et al. 2006). By only adopting two different temperatures (i.e., using a same dust opacity $k_{1.3}=0.5 \mathrm{~cm}^{2} \mathrm{~g}^{-1}$ ) we obtain a similar mass spectrum $(\alpha=1.44)$ but a less steep mass-size relation $\left(M \sim D^{1.38}\right)$, whereas two different temperatures and two different opacities yield both a steeper mass spectrum $(\alpha=1.89)$ and a shallower mass-size relation $\left(M \sim D^{1.12}\right)$.

However, the fraction of cores where dense warm dust dominates may be lower than envisaged above. See, e.g., Fig. 2: the cores MMS1, 2 and 3 are relatively far from the IRAS uncertainty ellipse, at the border of the HII region. Since the $1.2 \mathrm{~mm}$ emission from the area of the optical/radio HII region appears higher than extrapolated from the radio flux density (see previous section) assuming optically thin free-free emission, possibly there exists also warm dust mixed with the ionized gas, but this lies outside the cores. Consider the case of IRS17, as well: as discussed in Giannini et al. (2005), see also Fig. 3, the core found towards the IRAS source (MMS4) encompasses both the IRAS uncertainty ellipse and a less evolved protostar (at the origin of a prominent NIR jet). Then, although it was included in the subset of sources with higher temperature and dust opacity, it is actually possible that a major contribution comes from cold dust surrounding the protostar (i.e., with lower temperature and dust opacity). So, a large fraction of the $1.2 \mathrm{~mm}$ emission from IRS16 and IRS17 may be actually due to colder dust and this might be true for the whole subset of cores to which a higher temperature and dust opacity have been attributed in running this test. Nevertheless, the mass spectra and size-mass relations as derived in the two cases of uniform and different mixtures of environments probably bracket the actual ones.

A core mass spectrum with $\alpha \sim 1.4-1.9$ (or $\alpha \sim 1.2$ up to $\sim 40 M_{\odot}$ ) is consistent with the clump mass spectra in the same region obtained from $\mathrm{CO}(1-0)$ and ${ }^{13} \mathrm{CO}(2-1)$ by Elia et al. (2007), exhibiting spectral indices $\alpha \sim 1.3-2.0$. This may suggest a common origin for clumps and cores; however, Elia et al. (2007) discuss a number of problems in the derivation of the mass spectra from line observations and also find steeper mass-size relationships.

A "flat" mass-size relation has been shown to be consistent with a sample of pressure-confined Bonnor-Ebert condensations exhibiting uniform temperature and a range of external pressures (e.g., Motte et al. 2001; Johnstone et al. 2000). Whether the observed mass-size relation is due to all cores being close to the critical Bonnor-Ebert sphere relation, in which case $M \propto D$ for a range of pressures, or whether it is formed by families of Bonnor-Ebert spheres with varying importance of gravity, in which case $M \propto D^{3}$ for any fixed pressure, is uncertain at the sensitivity limits of present surveys. A mass-size relation $M \sim D^{2}$ would be more consistent with turbulent molecular cloud fragmentation producing a fractal distribution of core masses and sizes (e.g., Elmegreen \& Falgarone 1996). Actually, a sample of singular isothermal spheres (e.g., Shu et al. 1987) with constant sound speed and different masses would exhibit a $M \sim D$ relation, as well. However, this would imply that the clumps are all gravity bound, otherwise a much larger variation of the external pressure would be required to confine them with respect to Bonnor-Ebert condensations.

The mass-size relation we derive $\left(M \sim D^{1.7}\right)$ is shallower than that predicted for molecular cloud turbulent fragmentation, with the exponent in between those expected for the different families of Bonnor-Ebert spheres. However, we showed that the actual exponent is sensitive to the fraction of warm dense gas within the sample, decreasing with increasing this fraction. When crudely accounting for this effect, the exponent lies between 1.5 and 1, ruling out turbulent fragmentation and suggesting that our sample includes gas condensations in different evolutionary stages. High resolution measurements of the cores' column density profiles would be critical to clarify the issue.

The core mass spectrum between $\sim 1$ and $\sim 100 M_{\odot}$ is shallower than the stellar IMF in the same mass range, even in the worst case of a mixture of cores of different densities and temperature. Massi et al. (2006) find a standard stellar IMF for a sample of 6 young star clusters in the D cloud (5 of them associated with observed $\mathrm{mm}$ cores), hence the core mass spectrum is more reminiscent of the mass spectrum of molecular clumps, as already noted. This means that the larger cores 
underwent fragmentation, but this has not been observed at $\mathrm{mm}$ wavelength because of low resolution. The core mass spectrum should then be compared with the cluster mass spectrum (i.e., the mass distribution of stellar clusters) rather than the stellar IMF. Only at the lower mass end, the core mass spectrum may be dominated by the precursors of lower mass isolated stars and is likely to approximate an IMF. Remarkably, Lorenzetti et al. (1993), based on FIR fluxes from the IRAS Point Source Catalogue, derived a mass distribution with an index $\sim 1.5$ for the brightest protostars in the Class I stage associated with the Vela Molecular Ridge. As found by Massi et al. (1999), although the most luminous IRAS sources coincide with embedded young clusters, it is the most massive star of each cluster that appears to dominate the FIR emission. If so, the similarity between core mass spectrum and Class I source mass spectrum suggests that the mass of the most massive stars originated by a core is roughly proportional to the core's mass itself, at least at the high mass end. So, the core mass spectrum would reflect the mass spectrum of the most massive stars of every stellar group and, probably, the cluster mass spectrum, whereas the stellar IMF would be dominated by the fragmentation undergone by the single cores. Note that if the number of clusters' members and the mass of the most massive star in a cluster both depend on the mass of the parental core, then there must also exist an observable relationship between number of clusters' members and mass of the most massive star in a cluster. This would confirm that small stellar clusters cannot produce massive stars, explaining the possible lack of massive stars in the region as suggested by the results of the NIR observations by Massi et al. (2006).

It is straightforward to derive a gas density $n\left(\mathrm{H}_{2}\right) \sim 10^{5} \mathrm{~cm}^{-3}$ for MMS4, the most massive mm core. A mass-size relation $M \sim$ $D^{1.7}$ implies a density-size relation $n\left(\mathrm{H}_{2}\right) \sim D^{-1.3}$, so it is easy to check that the density of mm cores is $>10^{5} \mathrm{~cm}^{-3}$. We can estimate the fraction of gas mass in dense (i.e., $n\left(\mathrm{H}_{2}\right)>10^{5} \mathrm{~cm}^{-3}$ ) cores over the total gas mass by summing up all masses listed in Table 1; as a whole, we obtain $\sim 355 M_{\odot}$ of gas from $1.2 \mathrm{~mm}$ emission. Using our $\mathrm{CO}$ observations, the cloud mass ranges from $1.5 \times 10^{4} M_{\odot}$ (based on the ${ }^{12} \mathrm{CO}$ integrated emission) to $3.7 \times 10^{3} M_{\odot}$ (based on the LTE method and roughly correcting for the area not observed in ${ }^{13} \mathrm{CO}$ ), hence $\sim 2.4-10 \%$ of the gas lies in dense cores. The lower end is comparable, e.g., to the mass fraction of starless and prestellar cores of the most active star forming region in L1688 (the $\rho$ Oph. main cloud) and L1689, but is larger than the "total" mass fraction of starless and prestellar cores in the two clouds (Nutter et al. 2006). Johnstone et al. (2004) find that the submillimetre objects represent only a $2.5 \%$ of the mass of the Ophiuchus cloud. Kirk et al. (2006) surveyed the Perseus molecular cloud and derived a gas mass contained in submillimetre objects amounting to a $1 \%$ (or even less) of the total cloud mass, that seems consistent with the results of Hatchell et al. (2005). Hence, only few percent of the mass of molecular clouds appears to be in the form of dense cores and it is difficult to assess whether this mass fraction may be related with global properties of the parental clouds.

By comparing the total mass of dense gas associated with each embedded star cluster and the total stellar mass estimated by Massi et al. (2006), we find that in IRS16, IRS17 and IRS 21 the mass is almost equally distributed between dense gas and stars, whereas in IRS19 and IRS21 the stellar mass is larger than that of dense gas. Hence, for IRS16, IRS17, IRS19 and IRS21, the star formation efficiency computed by using $1.2 \mathrm{~mm}$ continuum emission ranges between 50 and $66 \%$, whereas that computed by using line emission (according to Massi et al. 2006) appears lower, 10-26\%. Obviously, the latter takes account of less dense gas not detected in the continuum. In the case of IRS20, in contrast, the star formation efficiencies are similar (80 and $77 \%$ ). Towards all of the 5 clusters, there are in principle still sufficiently massive cores to produce high-mass stars, provided a high efficiency is possible in converting dense gas into a star. Outside the clusters, massive cores have not been found and only intermediate- and low-mass stars can form.

An extinction threshold for star formation has been suggested by several authors. E.g., Onishi et al. (1998) and Johnstone et al. (2004) find $A_{\mathrm{V}} \sim 7-9$ mag in Taurus and Ophiuchus. As shown in Fig. 8, there is quite a good correspondence of $\mathrm{CO}(1-0)$ integrated intensity enhancements and $\mathrm{mm}$ cores, and $\mathrm{CO}(1-0)$ is a good tracer of diffuse gas. Then, an $A_{\mathrm{V}}$ can be estimated by converting $\mathrm{CO}(1-0)$ integrated emission into $N\left(\mathrm{H}_{2}\right)$, using the empirical relation of Elia et al. (2007). No mm peaks are detected at $A_{\mathrm{V}}<12 \mathrm{mag}$, which compares well with the values found in Taurus and Ophiuchus, given the uncertainty in the conversion of $\mathrm{CO}(1-0)$ integrated emission into $\mathrm{H}_{2}$ column density. However, we showed that our survey is probably not complete below $1 M_{\odot}$ and so low-mass cores may have escaped detection.

To assess the nature of the observed filaments, we attempted the analysis made by Hatchell et al. (2005), which is based on the work by Fiege \& Pudritz (2000). We considered the filaments composed of mm cores MMS5 and MMS6 (east of IRS17), MMS8 and MMS9, MMS10 and MMS11, MMS17 and MMS18, MMS19, MMS20 and MMS21 (close to IRS19). All have a small mass per unit length, ranging from $\sim 5$ to $\sim 40 M_{\odot} \mathrm{pc}^{-1}$. The virial mass entering Eq. (11) of Fiege \& Pudritz (2000) was derived through their Eq. (12) by using the $F W H M$ of ${ }^{13} \mathrm{CO}(2-1)$ lines observed towards the $\mathrm{mm}$ cores. The FWHM's span the interval $1.2-2.8 \mathrm{~km} \mathrm{~s}^{-1}$, increasing towards cores associated with IRAS sources (with the exception of MMS5 and MMS6, not associated with IRAS sources but exhibiting a large $F W H M$, i.e. $2.3 \mathrm{~km} \mathrm{~s}^{-1}$ ). The velocity dispersion $(\sigma)$ entering the virial mass has been determined using Eq. (20) and Eq. (21) of Fiege \& Pudritz (2000), assuming a gas temperature of $30 \mathrm{~K}$. We obtained virial masses $m_{\text {vir }}$ in the range $\sim 180$ to $\sim 500 M_{\odot} \mathrm{pc}^{-1}$, in all cases at least one order of magnitude more than the observed masses per unit length. Since the filaments lie well within the larger filaments of molecular gas, we derived the external pressure $P_{\mathrm{S}}$ on the cores using Eq. (2.12) of McKee (1989). Given the average column density is $\sim 10^{22} \mathrm{H}_{2} \mathrm{~cm}^{-2}$ towards the gas encompassing the denser filaments, the pressure over the cores' surface is $\sim 5 \times 10^{5} \mathrm{~K} \mathrm{~cm}^{-3}$. Assuming a density of $10^{5} \mathrm{~cm}^{-3}$, the cores' inner pressure is given by $P=\sigma^{2} \rho$, i.e., in the range $1-5 \times 10^{7} \mathrm{~K} \mathrm{~cm}^{-3}$. Following the analysis of Fiege \& Pudritz (2000), in particular with reference to their Eq. (11), the filaments cannot be confined by the external pressure and a toroidal magnetic field is needed to confine them. This is consistent with what found by Elia et al. (2007) for molecular clumps, i.e. the clumps' virial masses are always about an order of magnitude larger than the observed ones. That depends in turn on the derived velocity dispersion. However, the velocity dispersion as determined from ${ }^{13} \mathrm{CO}(2-1)$ is very likely not the correct one within the filaments. ${ }^{13} \mathrm{CO}(2-1)$ traces the lower density gas outside the dense cores and is probably much more sensitive to the star formation activity that is ongoing in the region. Hence, $\mathrm{mm}$ observations of high-density tracers and an assessment of the evolutionary status of the filaments through a comparison with near- and mid-IR data is necessary to analyse the stability of the filaments. In conclusion, the present data do not allow us to ascertain whether the fainter filaments are pressure- or magnetically confined, are collapsing structures or are dispersing. 


\section{Conclusions}

The discussion above suggests the following scenario for star formation activity in the observed molecular cloud. The arcs and double-peaked spectra evidenced by the $\mathrm{CO}(1-0)$ data suggest that the current gas distribution has its origin in intersecting expanding shells. The shells fragmented producing large filaments with embedded dense clumps. Within the clumps themselves, filaments of dense gas (less than $10 \%$ of the total gas mass) fragmented giving rise to the dense cores observed in the $\mathrm{mm}$ continuum. The most massive cores are associated with embedded young stellar clusters, indicating further fragmentation. The efficiency with which dense gas is converted into stellar mass appears high, $>50 \%$. Furthermore, the dynamical picture would confirm the widespread view according to which cluster formation is triggered by external compression of the parental gas (for a brief review, see Tan 2005). Massi et al. (2006) infer ages $>10^{6} \mathrm{yr}$ for the embedded clusters, which is consistent with the shells' dynamical age (see Elia et al. 2007). Hence, we are probably sketching star formation occurring in the last few Myr. The core mass spectrum appears flatter than a standard IMF in the range $\sim 1-100 M_{\odot}$, but agrees with the typical mass spectra of molecular clumps. Flat mass spectra have also been obtained from $\mathrm{CO}$ observations of the same region. It also agrees with the mass distribution of relatively luminous Class I sources in the region, indicating a continuity between molecular clumps, dense cores and the most massive stars in each cluster. The core mass spectrum should also be compared with the cluster mass spectrum in the region. Since, as found by Massi et al. (2006), the stellar IMF in the clusters appears similar to a standard IMF, it is likely that the "global" IMF results from the bulk of clusters' members, accounting for the apparent discrepancy between the cores mass spectrum and a standard IMF. Massi et al. (2006) find also a lack of high-mass stars in relation to what would be expected from a standard IMF. As pointed out by Lorenzetti et al. (1993), no massive star formation is currently occurring in the region. This would be the case if both the mass of the most massive member in a cluster and the number of clusters' members were roughly proportional to the mass of the parental cores, as suggested by the similarity between mass spectra. However, a few cores are massive enough to produce high-mass stars. To assess this possibility, further highresolution multi-wavelength observations are needed to delineate the actual evolutionary stage of the cores.

In summary, the main quantitative results are:

1. The core mass spectrum follows a relation $\mathrm{d} N / \mathrm{d} M \sim M^{-\alpha}$ between $\sim 1-100 M_{\odot}$, with $\alpha \sim 1.45-1.9$ depending on the temperature and opacity distribution within the sample; if a two-segments power-law is fitted, the power-law index is $\alpha=$ 1.2 up to $\sim 40 M_{\odot}$ and appears significantly larger beyond;

2. A mass-size relation $M \sim D^{x}$ has been derived for the cores, with $x \sim 1.1-1.7$, again depending on the temperature and opacity distribution within the sample;
3. No cores have been detected in areas with $A_{\mathrm{V}}<12$ mag, a possible confirmation of the existence of an extinction threshold for star formation;

4. The filaments of dense gas towards some of the cores cannot be confined by external pressure, but this result critically depends on having used the velocity dispersion as inferred from ${ }^{13} \mathrm{CO}(2-1)$ observations.

\section{References}

Beltrán, M. T., Brand, J., Cesaroni, R., et al. 2006, A\&A, 447, 221 Braine, J., Krügel, E., Sievers, A., \& Wielebinski, R. 1995, A\&A, 295, L55 Caswell, J. L., \& Haynes, R. F. 1987, A\&A, 171, 261

Chini, R., Kämpgen, K., Reipurth, B., et al. 2003, A\&A, 409, 235 De Luca, M., Giannini, T., Lorenzetti, D., et al. 2007, A\&A, submitted Elia, D., Massi, F., Strafella, F., et al. 2007, ApJ, 655, 316

Elmegreen, B. G., \& Falgarone, E. 1996, ApJ, 471, 816 Faúndez, S., Bronfman, L., Garay, G., et al. 2004, A\&A, 426, 97 Fiege, J. D., \& Pudritz, R. E. 2000, MNRAS, 311, 85

Giannini, T., Massi, F., Podio, L., et al. 2005, A\&A, 433, 941 Griffith, M. R., \& Wright, A. E. 1993, AJ, 105, 1666

Hatchell, J., Richer, J. S., Fuller, G. A., et al. 2005, A\&A, 440, 151 Heithausen, A., Bensch, F., Stutzki, J., Falgarone, E., \& Panis, J. F. 1998, A\&A, 331, L65

Johnstone, D., Wilson, C. D., Moriarty-Schieven, G., et al. 2000, ApJ, 545, 327 Johnstone, D., Boonman, A. M. S., \& van Dishoeck, E. F. 2003, A\&A, 412, 157 Johnstone, D., Di Francesco, J., \& Kirk, H. 2004, ApJ, 611, L45

Jørgensen, J. K., Johnstone, D., van Dishoeck, E. F., \& Doty, S. D. 2006, A\&A, 449, 609

Kerton, C. R., Martin, P. G., Johnstone, D., \& Ballantyne, D. R. 2001, ApJ, 552, 601

Kirk, H., Johnstone, D., \& Di Francesco, J. 2006, ApJ, 646, 1009

Liseau, R., Lorenzetti, D., Nisini, B., Spinoglio, L., \& Moneti, A. 1992, A\&A, 265,577

Lorenzetti, D., Spinoglio, L., \& Liseau, R. 1993, A\&A, 275, 489

Massi, F., Giannini, T., Lorenzetti, D., et al. 1999, A\&AS, 136, 471

Massi, F., Lorenzetti, D., Giannini, T., \& Vitali, F. 2000, A\&A, 353, 598

Massi, F., Lorenzetti, D., \& Giannini, T. 2003, A\&A, 399, 147

Massi, F., Testi, L., \& Vanzi, L. 2006, A\&A, 448, 1007

McKee, C. F. 1989, ApJ, 345, 782

Mitchell, G. F., Johnstone, D., Moriarty-Schieven, G., Fich, M., \& Tothill, N. F. H. 2001, ApJ, 556, 215

Mookerjea, B., Kramer, C., Nielbock, M., \& Nyman, L.-Ä. 2004, A\&A, 426, 119

Motte, F., André, P., \& Neri, R. 1998, A\&A, 336, 150

Motte, F., André, P., Ward-Thompson, D., \& Bontemps, S. 2001, A\&A, 372, L41

Murphy, D. C., \& May, J. 1991, A\&A, 247, 202

Nutter, D., Ward-Thompson, D., \& André, P. 2006, MNRAS, 368, 1833

Nyman, L.-Ä, Lerner, M., Nielbock, M., et al. 2001, ESO Messenger, 106, 40

Onishi, T., Mizuno, A., Kawamura, A., Ogawa, H., \& Fukui, Y. 1998, ApJ, 502, 296

Ossenkopf, V., \& Henning, Th. 1994, A\&A, 291, 943

Reid, M. A., \& Wilson, C. D. 2005, ApJ, 625, 891

Shu, F. H., Adams, F. C., \& Lizano, S. 1987, ARA\&A, 25, 23

Tan, J. 2005, Clustered Massive Star Formation in Molecular Clouds, in Massive Star Birth: a Crossroads of Astrophysics, ed. R. Cesaroni, M. Felli, E. Churchwell, \& M. Walmsley (Cambridge: Cambridge University Press), IAU Symp., 227, 318

Testi, L., \& Sargent, A. I. 1998, ApJ, 508, L91

Williams, J. P., de Geus, E. J., \& Blitz, L. 1994, ApJ, 428, 693

Yamaguchi, N., Mizuno, N., Saito, H., et al. 1999, PASJ, 51, 775 